\title{
BEAM INSTABILITY FOR LONGITUDINALLY INHOMOGENEOUS SYSTEMS
}

\author{
I. Litovko ${ }^{1}$, A. Goncharov ${ }^{2}$ \\ ${ }^{1} S C$ “Institute for Nuclear Research" NASU, 39, pr. Nauki, Kiev, Ukraine; \\ ${ }^{2}$ Institute of Physics NASU, 46, pr. Nauki, Kiev, Ukraine
}

PACS: 52.35.-g; 52.59.-f

\section{INTRODUCTION}

One of the main problems of ion beam physics is transport of the compensated ion beam in a plasma. Often for these processes the plasma parameters are not constant along the ion beam line. For example, if beam is focused - beam density and compensate charge particles density increase. If beam from different reason is expanded than his density and so compensate charge density is decreasing under the ion beam line. For such processes is a very important to find evolution of initial perturbations of beam particles in beam-plasma systems with inhomogeneous dialectical permittivity along their propagation.

In this article differential equation, describing spatial evolution of small perturbation for beam current density was obtained. It was made based on method presented firstly by Y. Fainberg and P. Blioch [1] for onecomponent beam. For some cases of beam-plasma systems analytical solutions this equation were founded. As an example computer simulations for transported ion beam and evolution of initial perturbation of plasma-beam instability on different parts of divided setup of isotopes electromagnetic separator were carried out.

\section{BASE EQUATIONS AND SOME ANALYTICAL SOLUTIONS}

We used linear two-liquid hydrodynamic model, describing space evolution for small fluctuations of nonuniform plasma-beam media. In [1] it was presented method, describing one component beam with changeable along propagation coordinate $x$ unperturbed velocity $V_{b}$ (but under $j_{b}=$ const), the method was developed in $[2,3]$ for two-component media. Follow by this method we can show that differential equation describing spatial evolution of small fluctuations of changeable beam current density on frequency $\omega$ has next form ( $V_{b}=$ const):

$$
j^{\prime \prime}(x)+j^{\prime}(x) M(x)+j(x) N(x)=0
$$

where:

$$
\begin{aligned}
& M(x)=\frac{2 i \omega L}{V_{0}}-\frac{d \ln n_{0}}{d x} \\
& N(x)=\frac{L^{2} \omega_{b}^{2}}{\varepsilon V_{0}^{2}}-\frac{L^{2} \omega^{2}}{V_{0}^{2}}-\frac{i \omega L}{V_{0}} \frac{d \ln n_{b}}{d x}
\end{aligned}
$$

Here $L$ - scale of inhomogeneity, $\varepsilon=1-\frac{\omega_{p}^{2}(x)}{\omega^{2}}$ dialectical permittivity of cold plasma. From (1) and (2) using substitution $j(x)=A \exp \left(-\frac{1}{2} \int M(x) d x\right) I(x)$ we can derive:

$$
I^{\prime \prime}(x)+I(x) f(x)=0
$$

where

$f(x)=\frac{L^{2} \omega_{b}^{2}}{\varepsilon V_{0}^{2}}-\frac{1}{4}\left(\frac{d \ln n_{b}}{d x}\right)^{2}+\frac{1}{2} \frac{d^{2} \ln n_{b}}{d x^{2}}$

So, if we know spatial dependences of stationary distribution for beam $n_{b}(x)$ and plasma $n_{p}(x)$ and determine boundary conditions for $j(0)$ и $j^{\prime}(0)$, we can obtain distribution of oscillation amplitude current density along beam propagation on different frequencies.

Functions $n_{b}(x)$ and $n_{p}(x)$ can obtain different forms and equation (3) for common case must solve by numerically. But for some separate cases analytical solutions equation (3) is possible to get. One observe for example some dependency for $n_{b}(x)$ and $\varepsilon(x)=1-\alpha n_{p}(x)$.

I. Let us observe case, when plasma distribution density stays constant but beam density change along perturbation direction. So, follow this $\varepsilon(x)=$ const. Let us describe beam concentration by function $n_{b}(x)=\frac{a}{(1+b x)^{2}}$, here $a$ and $b-$ constants that determining by special physical conditions. Note that such description of beam density is typical for some parts of ion beam in magnetic separator by isotope [4]. For this case from (3) and (4) one can derive:

$I^{\prime \prime}(x)+I(x) \frac{\beta a}{\varepsilon(1+b x)^{2}}=0$, where $\beta=\frac{L^{2} \omega_{b}^{2}}{V_{0}^{2}}$

(5)

Take into account initial condition $j(0)=j_{0}$ and $j^{\prime}(0)=0$ expression for space evolution of initial perturbations amplitude of beam current density $j(x)$ for frequency region where $\varepsilon \leq 0$ can be obtain:

$$
\begin{aligned}
& j(x)=\frac{j_{0} e^{\frac{-i \omega x}{V_{0}}}}{\sqrt{|1+b x|}}\left\{\begin{array}{l}
\left(1+\frac{1}{2 \gamma}+\frac{i \omega}{V_{0} \gamma b}\right)|1+b x|^{\gamma}+ \\
\left(1-\frac{1}{2 \gamma}-\frac{i \omega}{V_{0} \gamma b}\right)|1+b x|^{-\gamma}
\end{array}\right\} \\
& \text { here } \gamma=\sqrt{\frac{1}{4}+\frac{\beta a}{b^{2}|\varepsilon|}}
\end{aligned}
$$




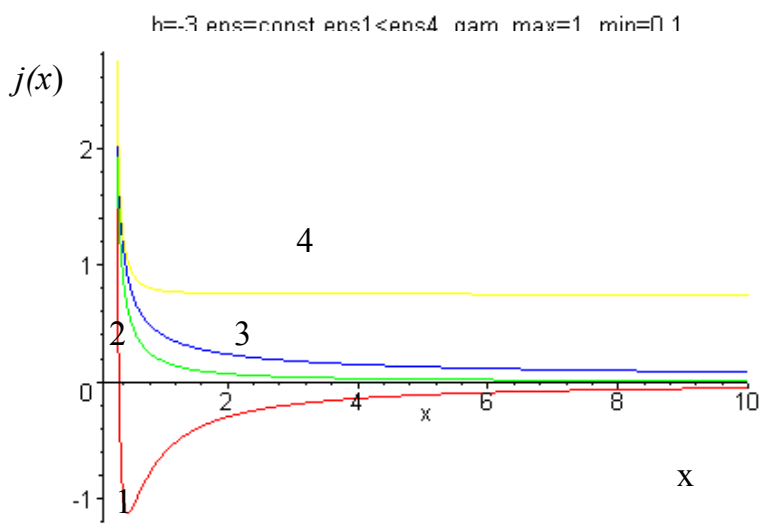

Fig 1. Dependence $j(x)$ on different $b<0, \varepsilon<0$ : 1) $\gamma=1$; 2) $\gamma=0.5$; 3) $\gamma=0.1$; 4) $\gamma=0.05$; $\varepsilon_{1}<\varepsilon_{4}$

Dependencies $j(x)$ had shown on figures 1 and 2. One can see that for $b>0$ (beam density decrease along propagation) current density amplitude grows very quickly and than faster then $|\varepsilon|$ smaller. For $b<0$, almost exponential grows for point $x=1 / b$, than fall down for point $x=2 / b$ and future stable near initial value $j_{0}$.

$j(x)$

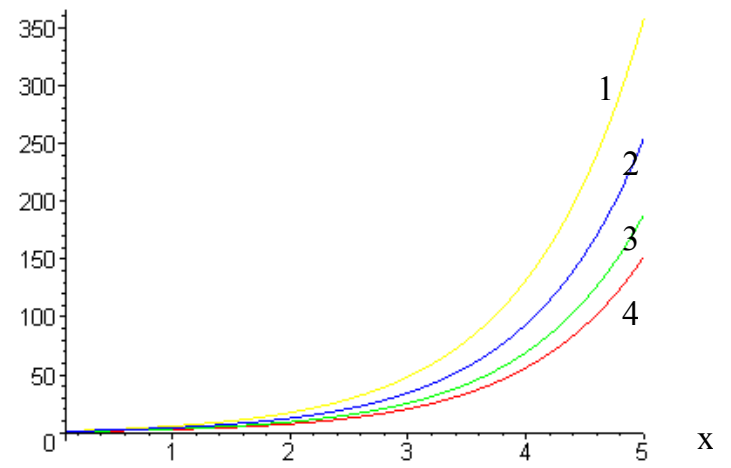

Fig 2. Dependence amplitude of current density under different $b>0$ and $\varepsilon<0$ : $1,3-b=1.5 ; 2,4-b=0.5 ;\left|\varepsilon_{1}\right|>\left|\varepsilon_{2}\right|$

II. Let us observe now case beam density and plasma particles density change both along propagation direction. This situation is typical for beam-plasma systems, in which plasma media is generating by beam.

Setting $n_{b}(x)=\frac{a}{(1+b x)^{2}}$ and $n_{b}(x) \sim n_{p}(x)$ one can derive equation (3) to hypergeometrical type:

$$
I^{\prime \prime}(x)+I(x) \frac{\beta a}{(1+b x)^{2}-\alpha a}=0
$$

here: $\beta=\frac{L^{2} \omega_{b}^{2}}{V_{0}^{2}}, \varepsilon(x)=1-\alpha n_{p}(x)$.

The solution can be obtain in form :

$$
\begin{aligned}
& j_{1}(x)=e^{-\frac{i \omega x}{V_{0}}}\left\{C_{1} \frac{1}{|1+b x|} F\left(\frac{\gamma}{2}-\frac{1}{4},-\frac{\gamma}{2}-\frac{1}{4} ; \frac{1}{2} ; z\right)+\right. \\
& \left.C_{2}\left(\frac{1}{\sqrt{\alpha a}}\right) F\left(\frac{\gamma}{2}+\frac{1}{4},-\frac{\gamma}{2}+\frac{1}{4} ; \frac{3}{2} ; z\right)\right\}, \quad \varepsilon(x)<0
\end{aligned}
$$

$$
\begin{aligned}
& j_{2}(x)=\frac{e^{-\frac{i \omega x}{V_{0}}}}{\sqrt{1+b x \mid \sqrt{\alpha a}}}\left\{C_{1}^{1}\left(\frac{(1+b x)}{\sqrt{\alpha a}}\right)^{-\gamma} F\left(\frac{\gamma}{2}-\frac{1}{4}, \frac{\gamma}{2}+\frac{1}{4} ; 1+\gamma ; \frac{1}{z}\right)\right. \\
& \left.+C_{2}^{1}\left(\frac{(1+b x)}{\sqrt{\alpha a}}\right)^{\gamma} F\left(-\frac{\gamma}{2}-\frac{1}{4},-\frac{\gamma}{2}+\frac{1}{4} ; 1-\gamma ; \frac{1}{z}\right)\right\}, \quad \varepsilon(x)>0 \\
& \text { where } \quad \gamma=\sqrt{\frac{1}{4}-\frac{\beta a}{b^{2}}}, \quad z=\frac{(1+b x)^{2}}{\alpha a}, \quad F(a, b, c, z)-
\end{aligned}
$$

hypergeometrical function. $\mathrm{C}_{1}, \mathrm{C}_{2}, \mathrm{C}_{1}{ }^{\prime}, \mathrm{C}_{2}{ }^{\prime}$ are constants that seeking from initial conditions $j(0)=j_{0}, j^{\prime}(0)=0$ and condition $\left.j_{1}(x)\right|_{\varepsilon=0}=\left.j_{2}(x)\right|_{\varepsilon=0}$. We are not present here these values from huge expressions. You can see transaction rom region with $\varepsilon<0$ to region with $\varepsilon>0$ for $b>0$ extension beam) on figures 3 . Current density amplitude _rows alike exponential for region $\varepsilon<0$. Dependence $j(x)$ on different $b>0$ in region $\varepsilon>0$ shown on Fig. 4 . One can see that for small $b(b<0.01)$ oscillations is fall dawn, but for $b^{n}$

$$
j(x)
$$

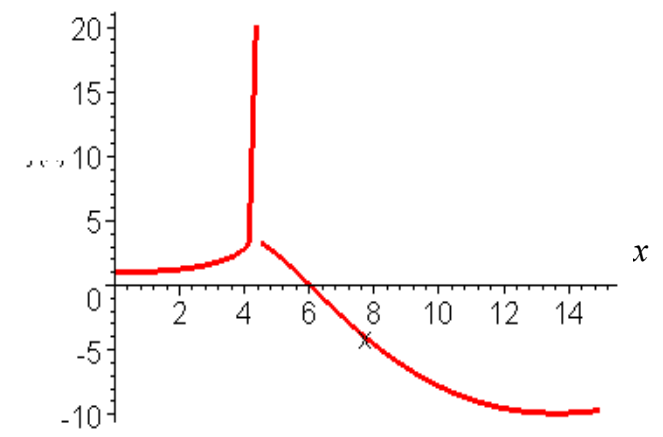

Fig 3. Transition from region with $\varepsilon<0$ to region with $\varepsilon>0$

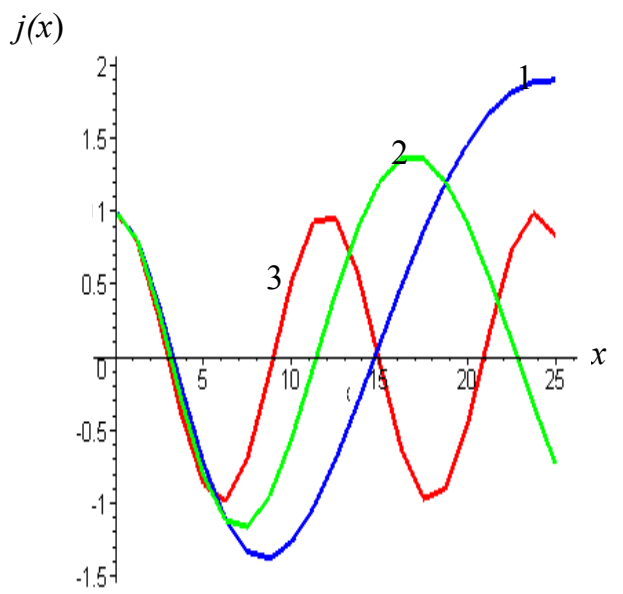

Fig 4. Behavior for extension beam $(b>0)$ in region with $\varepsilon>0 ; 1-b=0.1,2-b=0.01,3-b=0.005$

\section{COMPUTER SIMULATION PROPAGATION OF ION BEAM WITH VARIABLE DENSITY}

Equation (3) was solved by numerically for different parts of electromagnetic separator of isotopes. Electrons drift stabilizes potentials of low frequency noise oscillations along propagation direction of beam and decreases effect 
of dynamical decompensation and essentially influents on formation and transportation intensive ion beams in specific condition of electromagnetic separator of isotopes [4]. Essential role plays ion-electron instability that has maximal increment of increasing oscillations propagating along beam and can lead as to additional decompensation, as to energetic spectrum extension of separating beam. For calculation was take into account as ion-ion as ion-electron interactions. According this dialectical permittivity in beginning part of separator was described by next function:

$$
\begin{gathered}
\varepsilon(x)=1-\frac{\omega_{b}^{2}(0)}{\omega^{2}} \frac{f(x)}{2} n_{b}(x) \\
\text { where } n_{b}(x)=\frac{10}{\frac{5}{3} x+10} ; \quad f(x) \approx e^{-\frac{x}{4}} \text {. Thus equation }
\end{gathered}
$$

for beginning part of separator can present in form:

$$
I^{\prime \prime}+I\left(\frac{L^{2} \omega_{b}^{2}}{\varepsilon V_{0}^{2}}+\frac{1}{4} \frac{a^{2}}{(a x+b)^{2}}\right)=0
$$

here was set $a=5 / 3, b=10$.

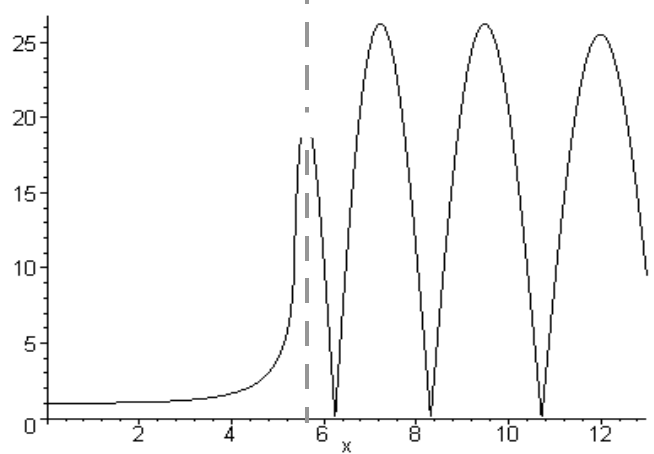

Fig. 5. Amplitude of current density for beginning part of separator for $\omega=0.5 \omega_{b}$ (dotted line separate region $\varepsilon$ $(x)<0$ from region with $\varepsilon(x)>0)$

For middle part dialectical permittivity changes and can describe by next function for ion-ion interactions:

$\varepsilon(x)=1-\frac{\omega_{b}^{2}(0)}{\omega^{2}} 3 \cdot 10^{-3} n_{b}(x)$, here

$n_{b}(x)=\frac{10}{\frac{5}{3} x+10-\frac{x^{2}}{21}}$. So equation for middle part

has form:

$$
I-f\left(\frac{L^{2} \frac{2}{b}+4 b c-t^{2}}{4\left(b-d x-e x^{2}\right)}=\theta\right.
$$

Here $a=5 / 3, b=10, c=1 / 21$.

For ion-electron interactions permittivity has form:

$$
\begin{aligned}
& \varepsilon(x)=1-0.9 \cdot 10^{3} \frac{\omega_{b}^{2}(x)}{\omega^{2}}\left(1+0.5 e^{-x}+0.03\right) \\
& \omega_{b}^{2}(x)=\frac{10}{10+\frac{5}{3} x} \omega_{b}^{2}(0,0)
\end{aligned}
$$

and equation follow:

$$
I^{\prime \prime}(x)+I(x)\left(\frac{L^{2} \omega_{b}^{2}}{\varepsilon V_{0}^{2}}+\frac{a^{2}}{4(a x+b)^{2}}\right)=0
$$

Results of calculation for begin part presented on figure 5. We can see that after transaction from region $\varepsilon$ $<0$ to region $\varepsilon>0$ oscillations can more increase. Results of calculation for middle with considering ion-ion and ion-electron interactions are on fig. 6. We can see that in middle part oscillations for some frequencies can significantly increase, especially for ion-electron interactions.
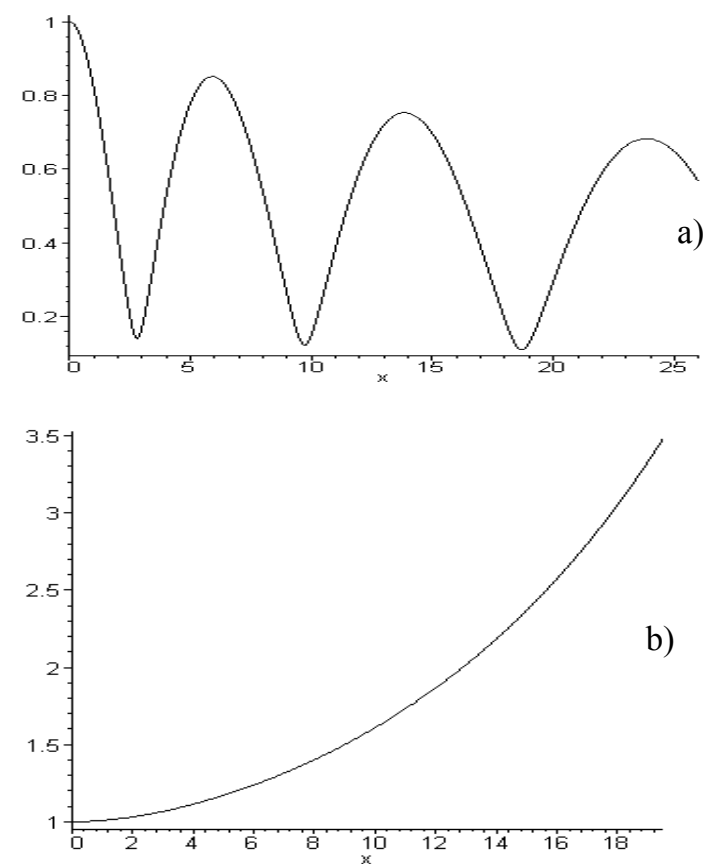

Fig. 6. Dependence $/ j(x) /$ for separator of isotopes for middle part with considering a) ion-ion interactions;

b) ion-electron interactions for $\omega_{b} / \omega=0.2$

\section{CONCLUSION}

Differential equation, describing spatial evolution of small perturbation of current density for beam-plasma system was obtained. It was shown that in some simple cases the equation could be solved analytically. For more common cases it can be solved using numerical methods. For expanded beam when it comes from region with negative dialectical permittivity to region with positive one essential rise of the beam current density oscillation take place. Experimentally these effects were observed for electromagnetic separator of isotope.

\section{REFERENCES}

1. P.V. Blioch, Y. B. Fainberg // Zhurnal Technicheskoy Fiziki 1956, v. 26, pp. 530-534, (in Russian).

2. M. I. Gechtman, A.A. Goncharov // Zhurnal Technicheskoy Fiziki 1990, v. 60, pp. 72-76 (in Russian).

3. A.A. Goncharov, I.V. Litovko//Proceedings of Conference on plasma accelerators and ion 
injectors. Kharkov, 1989, pp.155-156 (in Russian).

4. A.Goncharov, N.Datsko, I.Soloshenko // IEEE Trans. on Plasma Science, v. 23, \#5, 1995. 